\title{
Myocardial lysis in acute rheumatic fever followed by regeneration of cardiac muscle and origin of Aschoff bodies
}

\author{
HEMPROVA GHOSH MCDONALD
}

From the Diagnostic and Cell Research Institute, Waco, Texas, and Department of Surgical Pathology, Washington University School of Medicine, Saint Louis, Missouri

SYNOPSIS In acute rheumatic heart disease, lysis of cardiac muscle fibres with or without retention of sarcolemma is found to be the most damaging feature in many cases. In deeper myocardium the cellular lysis often forms anastomosing clefts or sinus-like spaces between surviving muscle bundles, and in the outer portion of myocardium cellular lysis may leave the sarcolemma more or less intact.

From lysing cardiac muscle fibres there arise dedifferentiated cells with remarkable potentiality for regeneration. For the origin of these dedifferentiated cells, which are often indistinguishable from lymphocytes, no mitosis is seen in cardiac muscle cells. The successive stages of development of muscle cells from these dedifferentiated cells within the remaining or newly formed sarcolemma have been observed in this study. This study infers that the increased number of fibrous septa, when seen, denotes the tracks of previous muscle degeneration and subsequent replacement of it with incomplete muscle regeneration and fibrous tissue formation.

In an area of muscle lysis the origin of Aschoff bodies from these dedifferentiated cells has been followed. Aschoff bodies arising in this way behave as an abortive and atypical growth of muscle fibres in a nodular fashion specific to rheumatic fever.

In the study of hearts of patients who died of acute rheumatic heart disease, degeneration as well as regeneration of myocardium is observed by following successive developmental changes as found in histological sections. In some cases myocardial lysis (liquefaction degeneration) is found to be the most prominent feature among the degenerative changes of the cardiac muscle. From lysing muscle cells, the origin of dedifferentiated cells (primitive cells in seed state) and their further development into cardiac muscle fibres will be described. Also Aschoff bodies, the diagnostic hallmark of rheumatic fever, are found to be a focal atypical growth of muscle fibres from these dedifferentiated cells.

The histological varieties of Aschoff bodies, as described by Gross and Ehrlich (1934), are believed to be related to different ways and phases of transformation of cardiac muscle cells into different types of cells including Anistchkow myocytes in the composition of Aschoff bodies (McDonald, 1957, 1962a, 1963). This paper will concentrate on myocardial lysis and the subsequent

Received for publication 15 January 1975. regeneration of cardiac muscle and Aschoff body formation.

\section{Material and Method}

Microscopic sections of all the hearts of patients who died at Barnes Hospital, Saint Louis, Missouri during a 38-year period with a diagnosis of rheu- $\rightarrow$ matic heart disease were studied. Excellent material for this study was obtained from the hearts of $N$ patients who died with acute rheumatic fever. New sections were made from such preserved heart $\mathcal{N}$ tissue fixed in Zenker-formol solution. In addition $\omega$ to the routine haematoxylin and eosin stain to study the degenerative and regenerative processes, the following stains were also used in some cases: Mallory's phosphotungstic acid haematoxylin, $\stackrel{?}{?}$ Mallory's aniline blue-acid fuchsin-orange $G, \frac{T}{O}$ Verhoeff van Gieson stain, Masson's trichrome $\frac{O}{\mathbb{D}}$ stain, Foot's modification of Bielschowsky's method $\frac{\rho}{\Phi}$ for reticulum stain, periodic acid Schiff method of $\stackrel{\complement}{\complement}$ McManus, and Benhold's Congo red stain. Serial sections were also studied when necessary to obtain $\varnothing$ three-dimensional views of particular structures. Both응 
conventional and phase microscopy were used; ill-defined cross striations were better identified by phase microscopy. A large number of photomicrographs have been critically studied in the histogenesis of rheumatic fever lesions of the myocardium.

The photomicrographs shown in this paper are taken from the ventricular wall of the following three acute rheumatic fever cases:

NECR OPSY 13489 (figs 1, 2, and 12)

On 11 April 1949 the fifth day of his second hospital admission, a 9-year-old boy died of acute rheumatic heart disease. Eighteen months before death he had developed joint and cardiac symptoms.

NECROPSY 6798 (figs 3-10)

A 17-year-old girl was admitted on 8 March 1937 because of swollen, inflamed ankle joints, fever of two weeks' duration, and anaemia. She died on the fourth day in hospital.

\section{NECROPSY 5283 (fig 11)}

An 11-year-old boy was admitted to hospital for the first time on 15 August 1932 with a diagnosis of acute rheumatic fever with congestive heart failure. He had two more hospital admissions before he died on 2 June 1933.

\section{Observations}

The study of acute cases of rheumatic heart disease presents demonstrable evidence that pathological changes, including the formation of Aschoff bodies of rheumatic fever, are due mainly to degeneration and reactive phenomena on the part of cardiac muscle fibres. The damage as well as repair of myocardium at their various phases is often observed in a single section and at times in a single microscopic field, giving an excellent opportunity for the study of the process of evolution. Observations presented in this paper may be divided into three broad categories: (1) damage of myocardium; (2) regeneration of cardiac muscle; and (3) origin of Aschoff bodies.

\section{DAMAGE OF MYOCARDIUM}

Of the various types of cellular changes resulting in damage of the myocardium, cellular lysis may be the most prominent feature in some cases and may involve a large portion of the cardiac muscle. Cellular lysis, although seen in scattered myocardial fibres or in isolated muscle bundles, usually forms wide anastomosing clefts or sinus-like spaces between the surviving muscle. An extreme case of such cellular lysis is shown (fig 1). Even at this low power one is able to see that some parts of the clefts are partially filled with a large number of Aschoff bodies; some of these Aschoff bodies are confluent. The extent of this cellular lysis will be more appreciated when one compares this figure with the relatively uninvolved part of the myocardium shown in the upper two-thirds of fig 2 , which was taken from a different microscopic field of the same section.

Since the edges of the cleft-like spaces formed by the surviving muscle soon become rounded off, and the cells inside the lumina are quickly liquefied once the liquefaction process has started, it is only by careful examination that one sees the evidence for lysis of cardiac muscle cells in producing these spaces obviously filled with fluid. Lysing cardiac muscle fibres with a faint sarcolemmic boundary can be seen inside the cleft in figure 4 .

The lysis of cardiac muscle in the outer portion of the myocardium usually leaves the sarcolemma more or less intact, and these sarcolemmae soon assume the spheroid shape shown in figs 11 and 12 . A more recent myocardial lysis with partial retention of the sarcolemma in a somewhat collapsed condition is seen in the broad mid-zone in fig 11 which is running parallel to the outer margin of the preserved myocardium. An Aschoff body can also be seen in this zone.

REGENERATION OF CARDIAC MUSCLE CELLS Even in acute fatal cases, regeneration of muscle fibres can be followed while lysis of cardiac muscle fibres is going on in neighbouring areas. Instead of mitosis for cell proliferation or maintenance of cell population, the lysing cardiac muscle cells give rise to primitive cells (dedifferentiated cells) with remarkable potential to rededifferentiate into muscle cells with cross striations. These dedifferentiated cells in 'seed state' are seen to arise within the muscle fibres showing various degrees of lysis. These cells arise as small hyperchromatic nuclei of round, oval or spindle shape; often multiple nuclei arise in a single muscle cell (fig 3). They soon lie in eccentric positions close to the cell margin of the mother cell; the normal nuclei of the muscle fibres by this time are usually no longer present. The cytoplasm of the mother cells may show varying degrees of change, that is, minimal to apparently complete lysis. These cellular changes in the muscle fibres are shown in figures 3 and 4 . The dedifferentiated cells in the early phase of cell development are almost naked of cytoplasm when they free themselves from the mother cells or when they lie within the preserved sarcolemma of the lysed mother cells.

In the process of regeneration of muscle fibres, where the original sarcolemma is no longer present, 


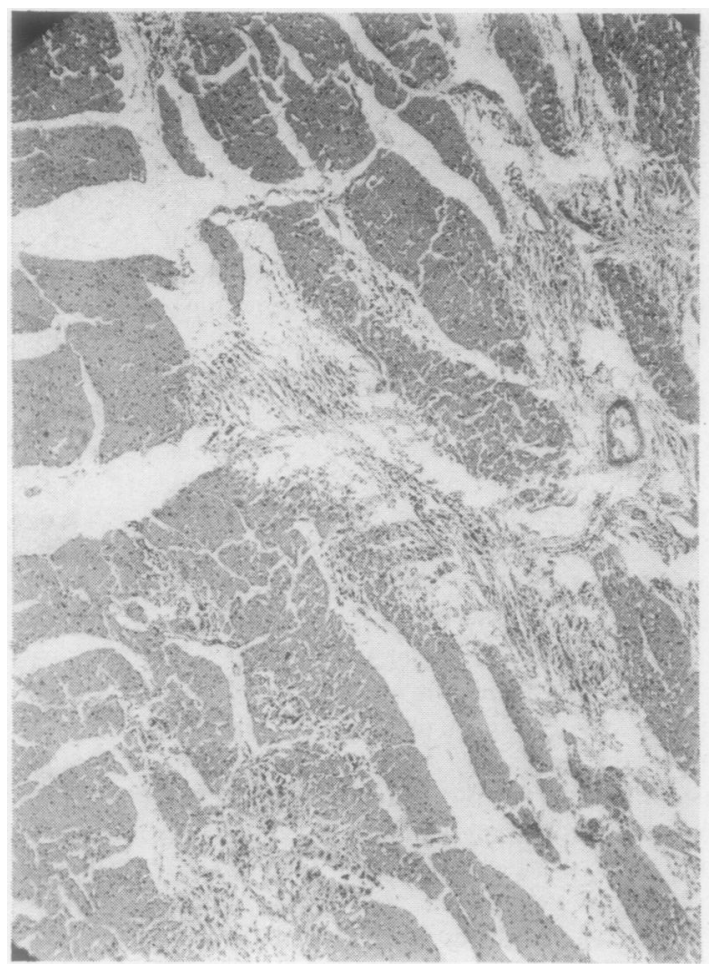

Fig 1

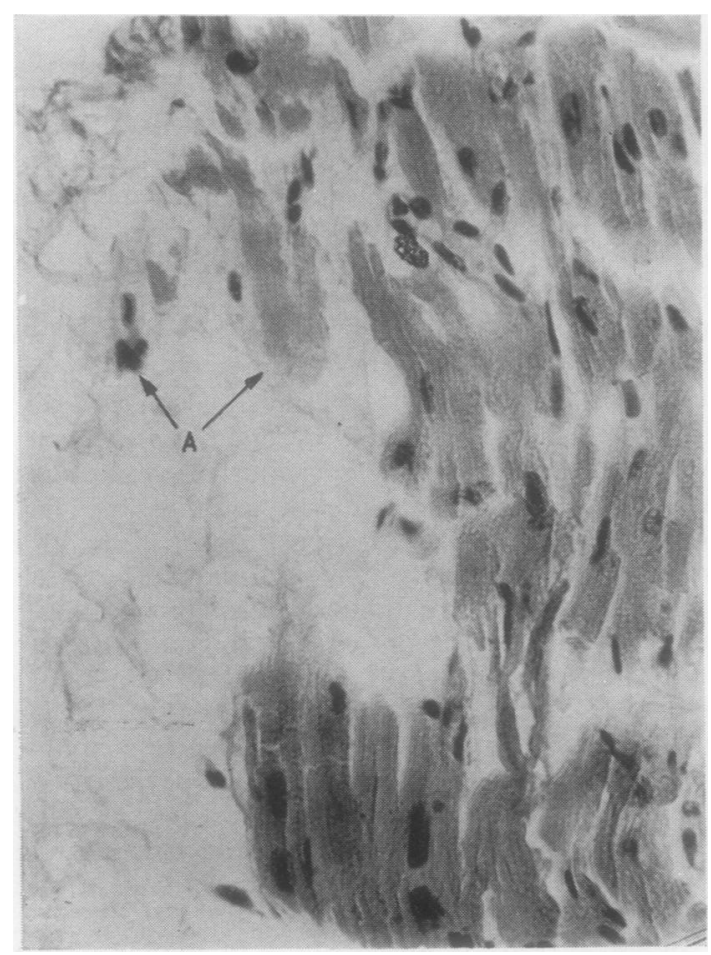

Fig 3

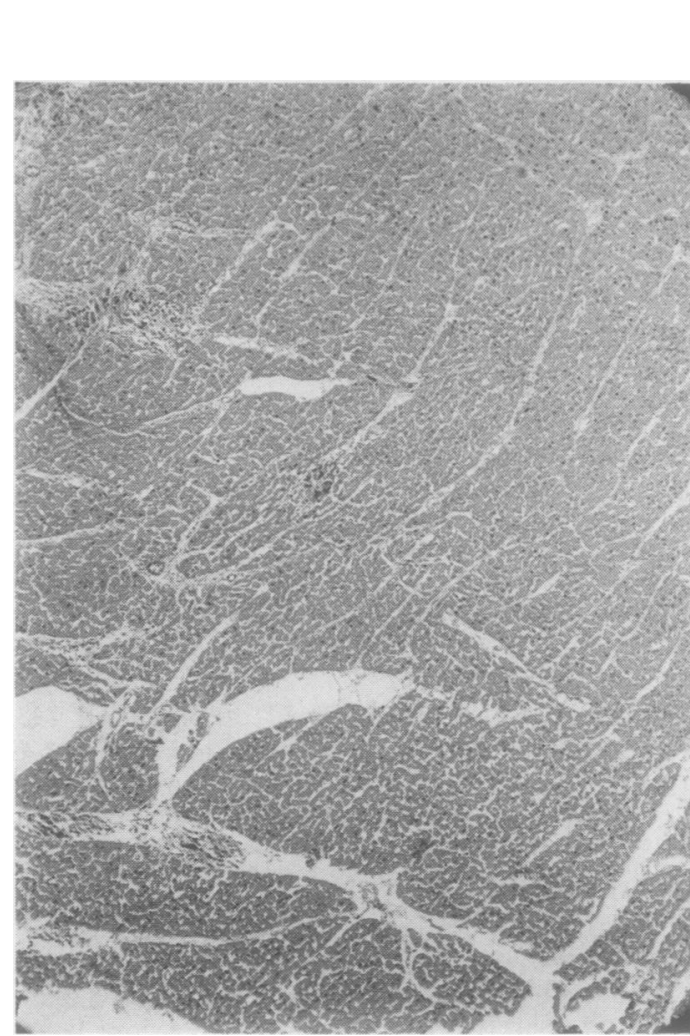

Fig 2

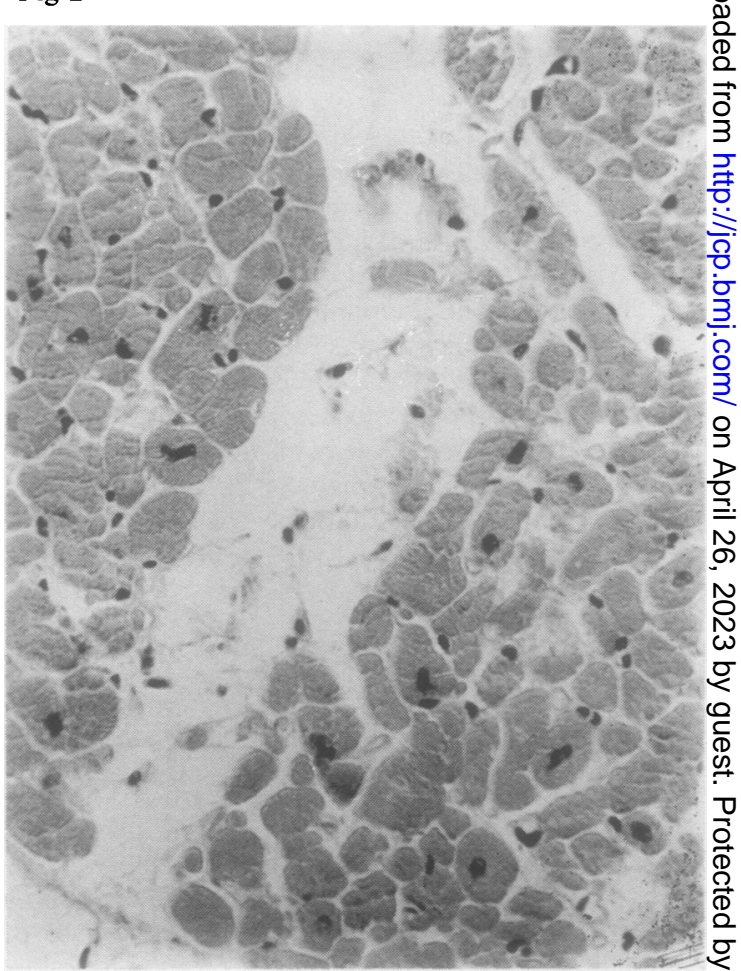

Fig 4 


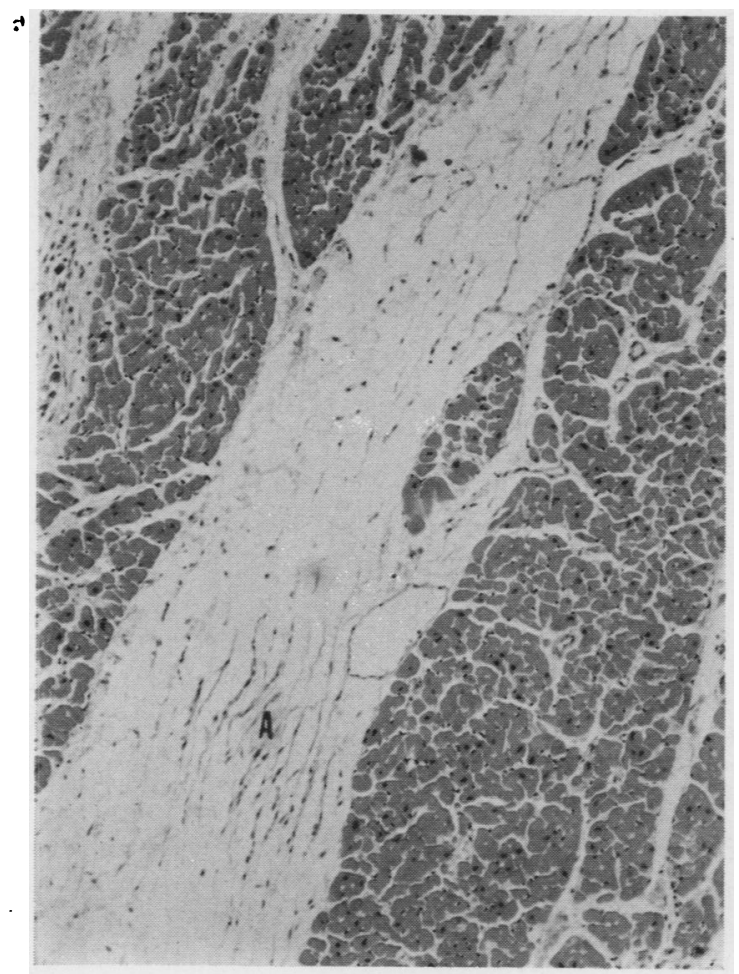

Fig 5

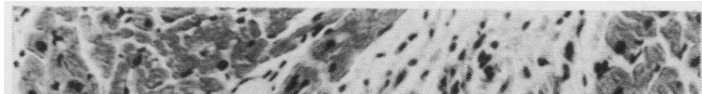

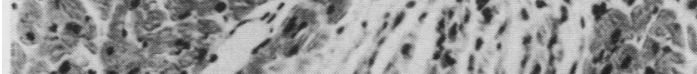

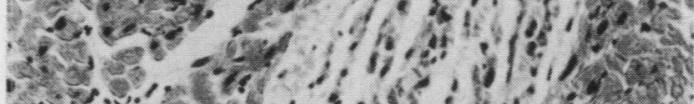
- 26.".

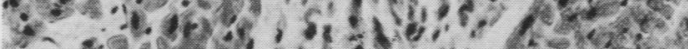

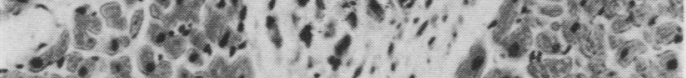

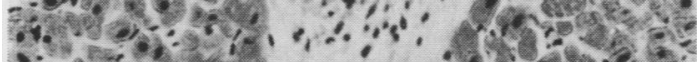
*at

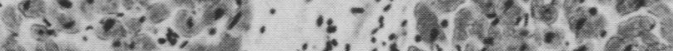
4.

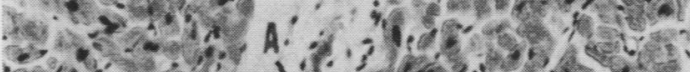

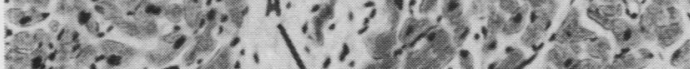

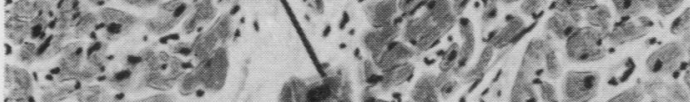
?

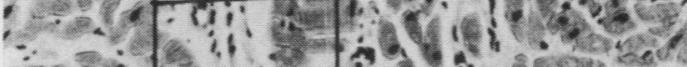

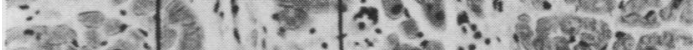

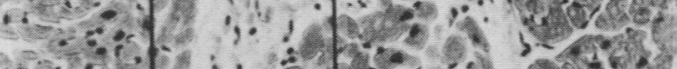

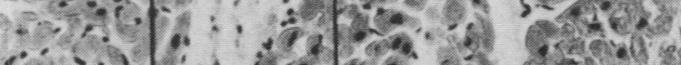

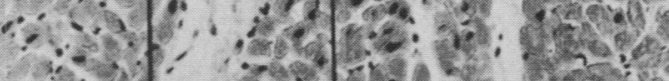

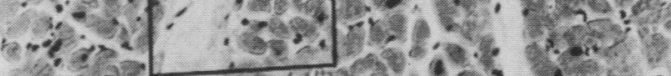

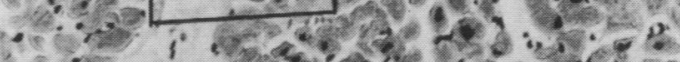

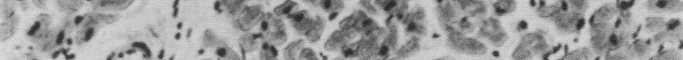

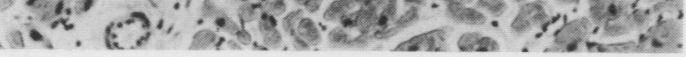

Fig 7

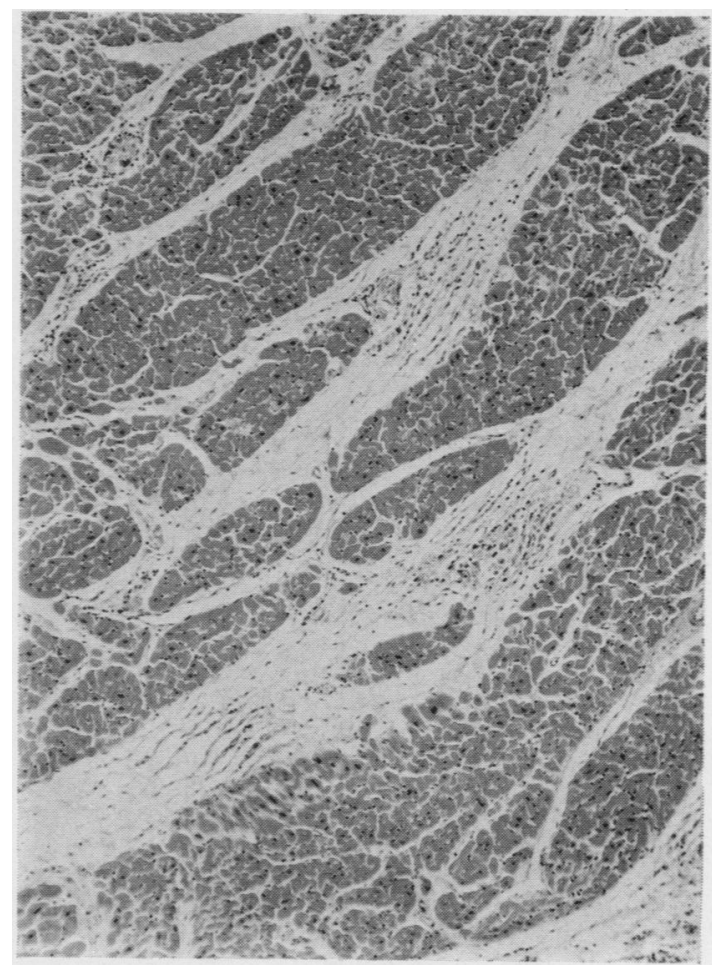

Fig 6

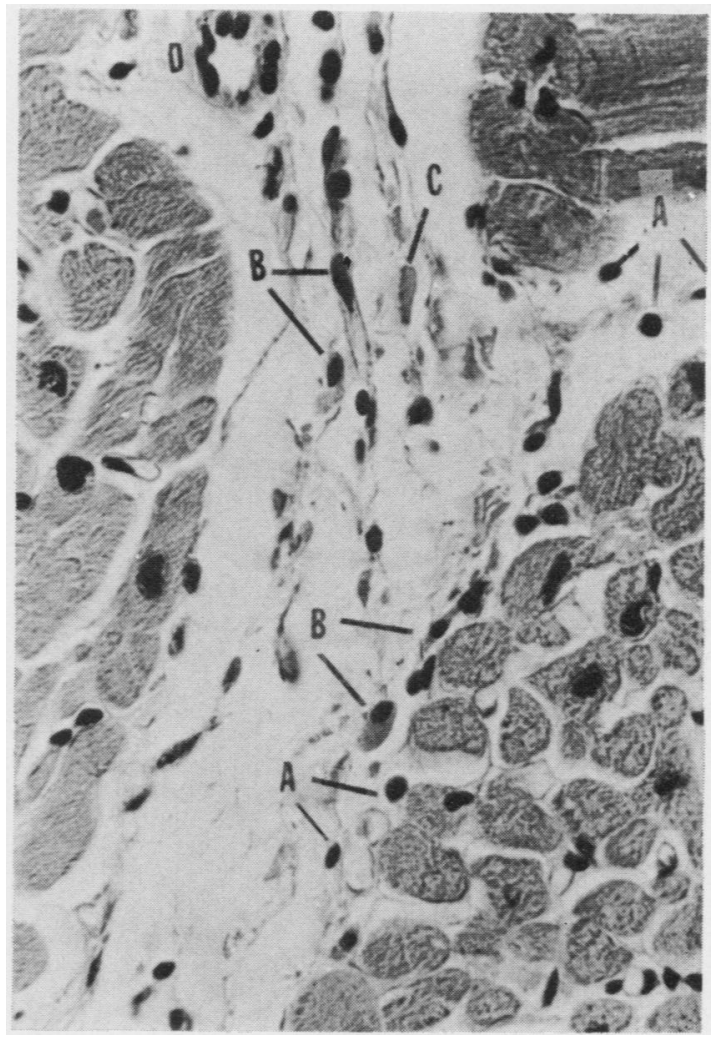

Fig 8 


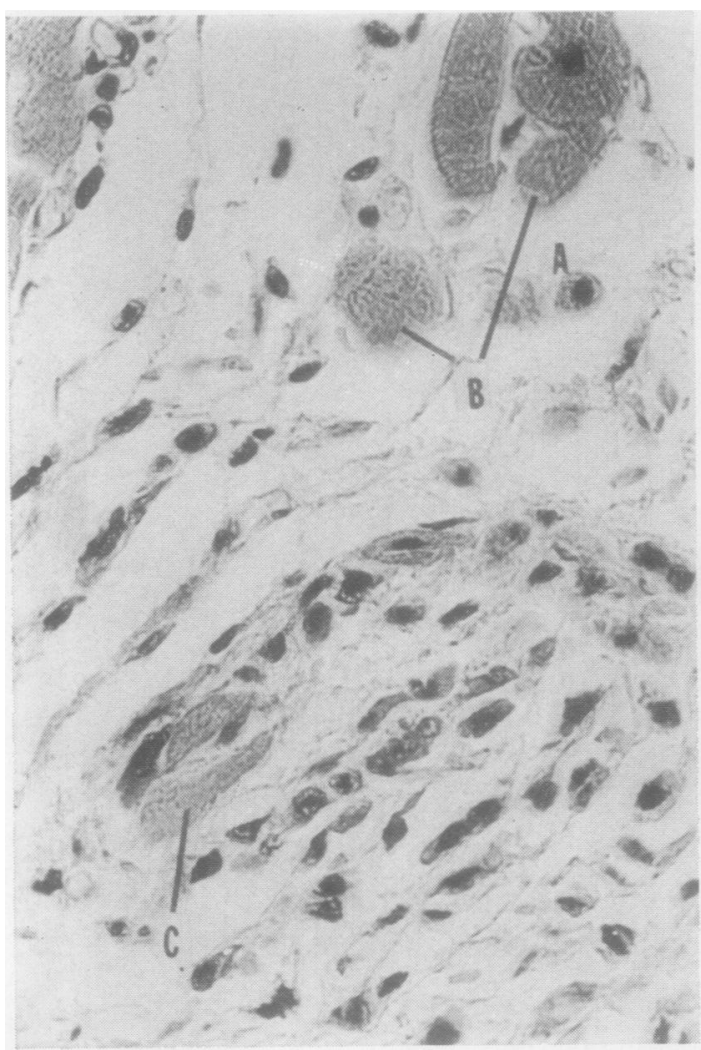

Fig 9

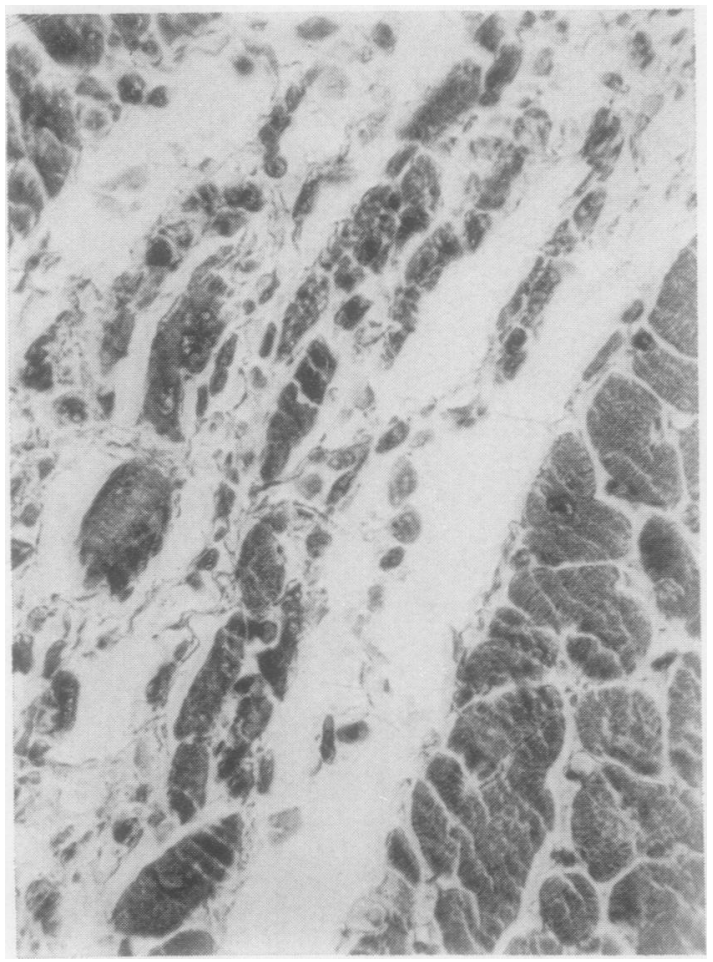

Fig 10

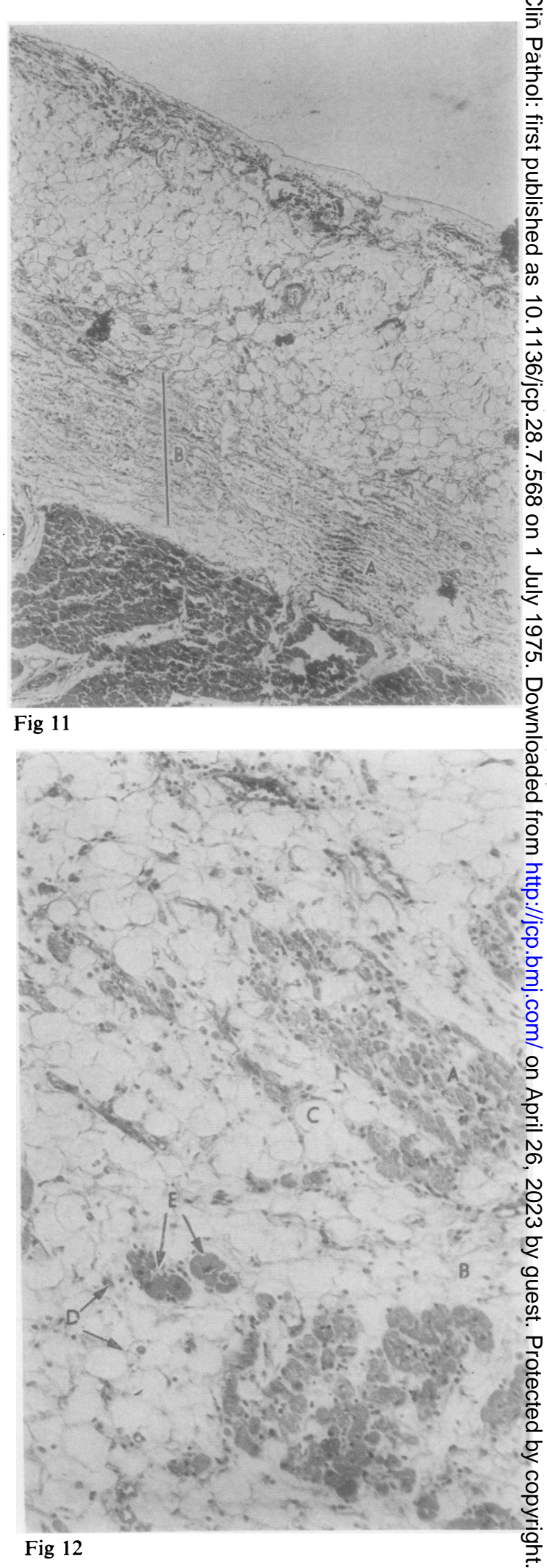


All photomicrographs are taken from ventricular walls of hearts of three patients (necropsy 5283, 6798, and 13489) who died of acute rheumatic heart disease. Figures 1, 2, and 12 are from necropsy 13489, figures 3 to 10 are from necropsy 6798 , and fig 11 is from necropsy 5283.

Fig 1 Extensive lysis of myocardium with formation of irregular clefts or sinus-like spaces and a large number of Aschoff bodies partially filling the lumina of these spaces. Haematoxylin and eosin $\times 40$

Fig 2 Photomicrograph taken within $2 \mathrm{~mm}$ of fig 1 , to give an idea of normal compactness of cardiac muscle as shown in the upper part of the field. Also demonstrates lysis of myocardium with formation of wide clefts and Aschoff bodies. $H$ and $E \times 50$

Fig 3 The myocardium near the epicardial surface is undergoing lysis. Note the origin of multiple, tiny, hyperchromatic dedifferentiated nuclei and the absence of normal cardiac muscle cell nuclei in lysing myofibres (A). Also note the disappearance of normal nuclei from many other muscle fibres. Sarcolemma of the lysed muscle cells is mostly faintly detectable here. $H$ and $E \times$ 335

Fig 4 Remnants of lysing cardiac muscle fibres and dedifferentiated cells with tiny hyperchromatic nuclei and with scarcely identifiable cytoplasm are lying in the cleft-like space formed by the lysis of muscle. Note also the similar nuclei, mostly in the peripheral portion of the less damaged myofibres in other areas. Compare with fig 3 for the origin, and with figs 5 to 12 for future development of these dedifferentiated cells. $H$ and $E \times$ 320

Fig 5 Preliminary stages of repair of the area of muscle lysis by dedifferentiated cells are shown. There is a delicate net-like formation by dedifferentiated cells preliminary to sarcolemma formation. A concentration of these fibrillary dedifferentiated cells in linear arrangement as the early stage of Aschoff body formation is seen in the left lower quadrant $(A)$. Two channel-like structures lined by dedifferentiated cells in a space of previous myocardial lysis can also be seen. $H$ and $E \times 78$

Fig 6 The successive stages of spindle-shaped Aschoff body formation are seen in the spaces derived from lysis of myocardium; see also figs 7-10. $H$ and $E \times 58$

Fig $7 \quad$ A spindle-shaped Aschoff body composed of Aschoff cells with shaggy cytoplasm, giving the appearance of what is known as fibrinoid, is shown in the upper part. The origin of cardiac muscle with cross striation as further development from this stage is shown in figs 9 and 10. Even in this Aschoff body under phase microscopy, cross striation is seen in a few Aschoff cells. The remaining area of the original pathway of muscle lysis becomes narrow due to the faster muscle regeneration process there, compared to the regenerative process within the Aschoff body. About an inch below the Aschoff body there is a newly formed group of muscle fibres; in one $(A)$ of these fibres, the full differentiation of nucleus into that of cardiac muscle cells can be seen. The enclosed area is explained in fig 8 . $H$ and $E \times 95$

Fig 8 Higher magnification of inset of fig 7. The same group of newly formed muscle fibres is shown in the upper left corner. $(A)$ dedifferentiated cells; $(B)$ myoblasts; (C) a segment of sarcoplasm with cross striation; and $(D)$ a small capillary-like structure lined by dedifferentiated cells. $H$ and $E \times 503$

Fig 9 In the process of repair of myocardial lysis, regeneration of muscle fibres within and outside the Aschoff body is shown. (A) a myoblast; (B) newly formed myofibres outside the Aschoff body; and $(C)$ myofibres within Aschoff body. $H$ and $E \times 450$

Fig 10 Differentiation of Aschoff cells into cardiac muscle fibres. Several muscle fibres with cross striations can be seen in this Aschoff body. Phosphotungstic acid-haematoxylin $\times 458$

Fig 11 Extensive myocardial damage involving the outer portion of the ventricular wall; the outer part of this photomicrograph simulates adipose tissue but the oblique mid-zone $(B)$ shows recent cellular lysis with partial retention of sarcolemma. In the outer and midzone are many dedifferentiated cells. A spindle-shaped Aschoff body formation in the mid-zone of cellular lysis is seen in the right lower quadrant $(A) . H$ and $E \times 49$

Fig 12 Subepicardial myocardium showing various phases of cellular lysis of muscle fibres with retention of sarcolemma and the origin of new muscle fibres within the retained sarcolemma by redifferentiation of dedifferentiated cells. (A) muscle fibres undergoing lysis; (B) an area from which sarcoplasm has been wiped out, but faintly stained sarcolemma and a few dedifferentiated cardiac cells are present (compare (B) with fig 3); (C) an area where the sarcolemma assumes a rounded appearance; $(D)$ myoblasts within retained sarcolemma; and $(E)$ bundles of newly formed cardiac muscle fibres. Compare the better staining quality and form of these newly formed muscle fibres with the degenerating muscle (A) and in other areas. $H$ and $E \times 49$ 
there appear fine fibrillary processes, many of which are connected with the dedifferentiated cells. A loose net-like structure formed by these fibrillary processes (fig 5) may act as the future sarcolemma as a preliminary need for orderly development of muscle. Regeneration of muscle fibres within the newly formed sarcolemma as further development of these dedifferentiated cells may be seen in figures 5-10. Dedifferentiated cells in the process of muscle formation are termed myoblasts (figs 8,9 , and 12 ).

It appears that full differentiation of cytoplasm with cross striation (sarcoplasm) is a rather sudden occurrence. Newly formed muscle fibres with cross striations stain sharply and strongly with special stains for muscle. The newly formed muscle cells within the retained sarcolemma, commonly seen in the outer myocardium, are shown in various stages of development(fig 12). Nucleardifferentiation from the dedifferentiated stage to that of typical cardiac muscle nucleus appears to be almost the last stage of cellular differentiation (fig 7).

Under favourable conditions the clefts or sinuses originally formed by muscle lysis may be completely or nearly closed by the newly formed muscle; this will be better appreciated by studying figures 5-10. Less than full measure of muscle regeneration may result in the formation of fibrous septa denoting the tracks of previous muscle lysis. The potential of these dedifferentiated cells to form connective tissue elements has also been observed (McDonald, 1957). Speidel (1938), in his experiment with skeletal muscle, observed similar dedifferentiated cells arising from skeletal muscle cells with the ability to form muscle and also connective tissue elements. Church (1970) and Mauro (1961) believed that 'satellite' cells (dedifferentiated cells) arising from skeletal muscle have the potential to form muscle fibres, and Church questioned the possibility of the 'satellite' cells transforming into fibroblasts and other cells in response to injury.

\section{ORIGIN OF ASCHOFF BODIES}

While new muscle formation is going on inside newly formed sarcolemma, for some unknown reason peculiar to rheumatic fever there appear concentrations of dedifferentiated cells with fibrillary processes arranged in somewhat parallel lines in spindle pattern ('A', fig 5) as an early stage of Aschoff body formation in the space created by previous muscle lysis. In further development of this structure, one can see the successive stages of Aschoff body formation in figures 6, 7, 9, and 10 . In the process of redifferentiation into cardiac muscle fibres, some of the dedifferentiated cells within Aschoff bodies acquire increasing amounts of cytoplasm, rather shaggy with ill-defined margins. These cells, known as Aschoff cells comprising the Aschoff bodies, are found to be cells in the process of regeneration of cardiac muscle fibres in a rather atypical way (figs 7, 9, and 10). The regenerating shaggy cytoplasm, before its attainment of cross striation, has the appearance of what has been known as fibrinoid material (figs 6-10). The nuclei of the regenerating muscle fibres attain their full development as cardiac muscle cell nuclei after the cytoplasmic differentiation with cross striation is apparently complete.

Regarding the descriptive classification of Aschoff bodies into seven types by Gross and Ehrlich (1934), one can see that several of these types are nothing but the different phases of Aschoff body formation as shown here. Other types of Aschoff bodies described by them are also found to be derived from cardiac muscle fibres undergoing a variety of degenerative changes but with less prominent cellular lysis (McDonald, 1957, 1962a, 1963).

It may also be pointed out that quite frequently one may see the large or small channels lined by dedifferentiated cells, as in figs 2 and 5 , in the cleft-like spaces created by muscle lysis. I have studied the possibility of vascular channels originating from these initial stages; the process has been similar to that seen in other tissues (McDonald, 1962b, 1968).

\section{Discussion}

Acute myocardial damage by cellular lysis, as has been demonstrated in this study, appears to be a direct effect of the injurious agent or a result of specific reaction associated with the rheumatic fever disease process. The loss of continuity of the heart muscle due to extensive lysis of the myocardium may explain, at least in part, the blockage in the conduction system giving rise to different types of arrhythmias.

It appears that myocardial lysis, as extensive as it is shown here, had been discarded in the past as artefact or oedema. The collection of a large amount of fluid in the myocardium without evidence of compression of adjacent muscle fibres led me to realize that replacement of the original tissue by fluid must have occurred; this process is in contrast to oedema formation in the serous cavities, lung, or areolar tissue. It is interesting to note that the experimental study of Menne, Jones, and Jones (1934) showed cellular changes (what has here been termed as lysis) of the myocardium in rabbits caused by increasing the heart rate mechanically and induced hyperthyroidism. It would be interest- 
ing to study the process of regeneration of new muscle fibres as a follow-up of their experiment.

The hopeful findings of this present study are the recognition of all-out attempts to repair the myocardial damage by regeneration of cardiac muscle. Probably myncardial regeneration has been overlooked in the past because mitosis does not appear to be the way by which the cardiac muscle normally regenerates. Mitosis in cardiac muscle has been reported in young rats in cases of experimental burn by Robledo (1956). I have not seen mitosis in normal cardiac muscle cells but have seen mitosis of Anitschkow myocytes. The process of cellular changes in the origin of Anitschkow myocytes from cardiac muscle fibres, as described by Anitschkow in 1913, has been observed (McDonald, 1963).

The dediffer $\epsilon$ tiated cells noted by Speidel (1938) in skeletal muscle and by me in cardiac muscle show the similarity of their origin and transformation into muscle fibres of respective orders. The dedifferentiated cells that Speidel found in skeletal muscle are similar to what have more recently been termed 'satellite cells' by Mauro (1961) and Church, Noronha, and Allbrook (1966) with a remarkable potential for regeneration of skeletal muscle in case of injury.

In addition, in agreement with Speidel, I observed the potential of these dedifferentiated cells to be transformed into connective tissue cells; this is seen particularly in the area of incomplete muscle replacement by formation of connective tissue septa denoting the landmark of previous myocardial lysis. The presence of unresolved Aschoff bodies along these connective tissue septa may have been interpreted as a support for mesenchymal origin of Aschoff bodies. It is worthwhile to note that Krösing (1892), after an extensive study, came to the conclusion that connective tissue may be formed as a result of retrogressive or anaplastic change in both skeletal and cardiac muscle and that this new connective tissue may form cicatrix and fat tissue.

The cardiac muscle cell origin of the Aschoff body has been demonstrated by Murphy (1952, 1959) and Whitman and Eastlake (1920); however, the extent of myocardial lysis and the remarkable potential for regeneration of cardiac muscle fibres in acute rheumatic fever have not previously been appreciated.

I express my grateful thanks to Dr Lauren V. Ackerman, formerly professor of surgical pathology,
Washington University School of Medicine for his encouragement in this study, and to my husband, Dr Hendley A. McDonald, for assistance in the preparation of this manuscript.

\section{References}

Anitschkow, N. (1913). Über die Histogenese der Myokardveränderungen bei einigen Intoxikationen. Virchow Arch. path. Anat., 211, 193-237.

Church, J. C. T. (1970). Cell populations in skeletal muscle after regeneration. J. Embryol. exp. Morph., 23, 531-537.

Church, J. C. T., Noronha, R. F. X., and Allbrook, D. B. (1966). Satellite cells and skeletal muscle regeneration. Brit. J. Surg., 53, 638-642.

Ghosh, Hemprova. See McDonald, Hemprova Ghosh.

Gross, L. and Ehrlich, J. C. (1934). Studies on the myocardial Aschoff body. I. Descriptive classification of lesions. Amer. J. Path., 10, 467-488.

Krösing, R. (1892). Ueber die Rückbildung und Entwickelung der quergestreiften Muskelfasern. Virchow Arch. path. Anat., 128, 445-484.

McDonald, Hemprova Ghosh (1957). Observations on the histogenesis of rheumatic lesions of the heart. (Abstr.) Amer. J. Path., 33, 598-599.

McDonald, Hemprova, Ghosh (1962a). Myocardial lysis and regeneration of cardiac muscle fibers through the stages of redifferentiation of dedifferentiated cells arising from cardiac muscle fibers as shown in acute rheumatic heart disease (Abstr.). Anat. Rec., 142, 257.

McDonald, Hemprova, Ghosh (1962b). Formation of new vascular channels from local tissues by cellular lysis as shown in mammary carcinomas in mice. J. Ind. med. Ass., 39, 115-123.

McDonald, Hemprova Ghosh (1963). Origin of Anitschkow's myocytes from cardiac muscle fibers. Texas St. J. Med., 59, 1062-1067.

McDonald, Hemprova Ghosh (1968). Origin of vascular channels from epithelial tissue as shown in livers. $J$. Amer. med. Wom. Ass., 23, 545-553.

Mauro, A. (1961). Satellite cell of skeletal muscle fibers. J. biophys. biochem. Cytol., 9, 493-495.

Menne, F. R., Jones, O. N., and Jones, N. W. (1934). Changes in the myocardium of rabbits from augmenting the heart rate mechanicelly and from induced hyperthyroidism. Arch. Path., 17, 333-355.

Murphy, G. E. (1952). Evidence that Aschoff bodies of rheumatic myocarditis develop from injured myofibers. J. exp. Med., 95, 319-332.

Murphy, G. E. (1959). On muscle cells, Aschoff bodies, and cardiac failure in rheumatic heart disease. Bull. N.Y. Acad. Med., 35, 619-651.

Robledo, M. (1956). Myocardial regeneration in young rats. Amer. J. Path., 32, 1215-1239.

Speidel, C. C. (1938). Studies of living muscles: growth, injury, and repair of striated muscle, as revealed by prolonged observations of individual fibres in living frog tadpoles. Amer. J. Anat., 62, 179-235.

Whitman, R. C. and Eastlake, A. C. (1920). Rheumatic myocarditis: a histogenic study of the type of cells of the Aschoff body. Arch. intern. Med., 26, 601-611. 